\title{
Loss of Pax6 Causes Regional Changes in DII1 Expression in Developing Cerebral Cortex
}

\author{
Elena Dorà ${ }^{\dagger}$, David J. Price and John O. Mason* \\ Centre for Discovery Brain Sciences, University of Edinburgh, Edinburgh, United Kingdom
}

The transcription factor Pax6 controls multiple aspects of forebrain development. Conditional deletion of Pax 6 in embryonic mouse cortex causes increased proliferation of cortical progenitor cells and a concomitant decrease in neural differentiation. Notch signaling regulates the balance between proliferation and differentiation of cortical progenitor cells, suggesting a possible connection between Pax6 and Notch signaling. We investigated how expression of the Notch ligand delta-like 1 (DI/1) is altered by loss of Pax6. Acute cortex-specific deletion of Pax6 resulted in a widespread decrease in the density of DII1+ cells at embryonic days 12.5 and 13.5 (E12.5 and E13.5). In constitutive

OPEN ACCESS

Edited by:

Satoshi Goto,

Tokushima University, Japan

Reviewed by:

Gonzalo Alvarez-Bolado,

Universität Heidelberg, Germany

Se-Young Choi,

Seoul National University,

South Korea

*Correspondence:

John O. Mason

john.mason@ed.ac.uk

tPresent address:

Elena Dorà,

Centre for Inflammation Research, University of Edinburgh, Edinburgh, United Kingdom

Received: 20 December 2018 Accepted: 15 February 2019

Published: 06 March 2019

Citation:

Dorà E, Price DJ and Mason JO (2019) Loss of Pax6 Causes Regional Changes in Dll1 Expression in Developing Cerebral Cortex. Front. Cell. Neurosci. 13:78. doi: 10.3389/fncel.2019.00078 loss-of-function mutants, decreases in the densities of DIl1 + cells were more limited both spatially and temporally. Controlled over-expression of Pax6 had no detectable effect on D/l1 expression. The proneural transcription factor Neurog2 is a target of Pax6 that can activate D/l1 expression and we found clear co-expression of Neurog2 and D/l1 in radial glial progenitors, suggesting that Pax6's effect on D/l1 could be mediated through Neurog2. However, we found no change in Dll1 + cells in Neurog2-/- cortex suggesting either that Neurog2 is not directly involved, or that its loss of function in embryonic cortex can be compensated for.

Keywords: Pax6, cortical development, progenitor cells, delta-like 1, Notch pathway

\section{INTRODUCTION}

As the brain develops during embryogenesis it must ensure that appropriate numbers of cells are born at the appropriate time, such that each region of the brain reaches the correct final size. To achieve this, the relative rates of proliferation and differentiation of neural progenitor cells must be tightly controlled. The earliest progenitor cells in the embryonic cortex, neuroepithelial cells, divide symmetrically to generate two new progenitors, expanding the progenitor pool. As development proceeds, neuroepithelial progenitors transform into radial glia cells (RGCs), a major class of neural progenitors. RGCs may divide symmetrically to give rise to two identical progenitor cell daughters, or asymmetrically, giving rise to one radial glial cell and one more differentiated cell-either a neuron, or an intermediate progenitor cell (IPC). In the mouse, most IPCs divide symmetrically to produce two neurons, but a small proportion divide asymmetrically to give rise to one progenitor and one neuron (Haubensak et al., 2004; Noctor et al., 2004; Pontious et al., 2008). Clearly, RGCs must carefully balance their output between neurogenic cells (IPCs and neurons) and new RGC progenitors. If too many neurogenic cells 
are produced early, this would lead to a reduction in progenitor numbers and an overall smaller cortex. Conversely, too few neurogenic divisions would increase progenitor numbers and cortical size.

The transcription factor Pax6 is a key regulator of forebrain development, with diverse roles in corticogenesis, including control of cortical cell number (reviewed by Manuel et al., 2015; Ypsilanti and Rubenstein, 2016). Pax6 is expressed highly in RGCs, but its levels decrease in IPCs (Elsen et al., 2018). We recently showed that acute deletion of Pax6 in the embryonic cortex leads to both an increase in cortical progenitor proliferation and a decrease in neural differentiation (Quintana-Urzainqui et al., 2018). This suggests that Pax6 can regulate the output from cortical RGCs by influencing the balance between their proliferation and differentiation. As a transcription factor, Pax6's effects on RGC output are most likely exerted through control of target gene expression. Many Pax6 regulated genes have been identified (Sansom et al., 2009; Wolf et al., 2009; Mi et al., 2013; Xie et al., 2013; Sun et al., 2015; Quintana-Urzainqui et al., 2018) including the pro-neurogenic transcription factor Neurog2 (previously known as Ngn2), which is directly regulated by Pax6 (Scardigli et al., 2003). In addition to its pro-neurogenic activity, Neurog2 activates expression of the Notch ligand Delta-like 1 (Dll1; Castro et al., 2006; Shimojo et al., 2008). Notch signaling is a well-characterized regulator of neural progenitor maintenance and differentiation. When Notch signaling is active, the Notch ligands Dll1 and Jagged engage Notch receptors on the surface of neighboring cells and induce expression of genes that inhibit neural differentiation, thereby maintaining the cell in a proliferative state (Pierfelice et al., 2011). Taken together, this suggests a plausible hypothesis to explain how Pax6 could influence behavior of the RGC progenitor pool in embryonic cortex. Pax6 activates Neurog2 expression in RGCs, in turn Neurog2 acts both to promote a pro-neural fate in cells expressing it and to drive expression of Dll1, which could then feed back to neighboring cells to promote their proliferation and thereby reduce their likelihood of becoming pro-neurogenic. Consistent with this idea, microarray and transcriptome sequencing studies have shown that overall levels of Dll1 expression are significantly decreased in $\mathrm{Pax6}^{-/-}$mutant cortex (Mi et al., 2013; Quintana-Urzainqui et al., 2018) and increased in transgenic mice that overexpress Pax6 (Sansom et al., 2009), but it is not known how the cellular pattern of Dll1 expression is affected in the absence of Pax6. Pax6 is expressed in a gradient across the developing cortex and some of its effects on cell cycle have been shown to be confined to regions where Pax6 expression is highest (Mi et al., 2013). It is therefore important to examine the spatial effects of Pax6 loss on Dll1 expression in more detail.

In the present study, we investigated how the spatial distribution of Dll1-expressing cells is affected by loss of Pax6. We found significantly reduced densities of Dll1-expressing cells in areas of the embryonic cortex in both constitutive and conditional Pax6 mutants, consistent with the suggestion that Pax6 promotes Dll1 expression. Interestingly, the effect on Dll1 expression was greater in conditional Pax6 mutants than in $\mathrm{Pax}^{-/-}$null mutants of the same age, suggesting the presence of a compensatory mechanism which has not had time to take effect in the conditional mutants. We also found that many RGCs co-expressed Neurog2 and Dll1, as predicted by our hypothesis. However, while loss of Pax6 led to a dramatic decrease in Neurog2 expression, its effect on Dll1 expression was less pronounced and Dll1 expression was unaffected in Neurog $2^{-/-}$mutant cortex, suggesting that additional levels of control of Dll1 expression are present.

\section{MATERIALS AND METHODS}

\section{Mice}

All animal experiments were conducted in accordance with the guidelines of the UK Animals (Scientific Procedures) Act 1986 and were approved by Edinburgh University's Animal Ethics Committee. For all crosses, the morning on which the vaginal plug appeared was deemed embryonic day 0.5 (E0.5). Pax6 ${ }^{-/-}$embryos were obtained by crossing Pax6 ${ }^{\text {Sey/+ }}$ heterozygotes (Hill et al., 1991) and non-homozygous littermates were used as controls. Pax6 cortex-specific conditional mutant embryos (Emx1cre-ER $R^{T 2} ;$ Pax $6^{\text {loxP } / \text { loxP }}$, abbreviated to $\left.\mathrm{KKO}\right)$ were obtained as described previously (Mi et al., 2013). Cre activity was induced by administering $10 \mathrm{mg}$ of tamoxifen (Sigma) by oral gavage at E9.5. PAX77 mice (Schedl et al., 1996) carry a yeast artificial chromosome transgene containing multiple copies of human PAX6 under the control of its own regulatory elements and express PAX6 protein at approximately three-fold of normal levels (Manuel et al., 2007). Neurog $2^{-/-}$embryos were obtained by crossing Neurog $2^{\text {cre/+ }}$ heterozygotes in which insertion of cre coding sequence creates a null allele of Neurog2 (Zirlinger et al., 2002). Embryos were dissected in cold phosphate buffered saline (PBS), fixed overnight in $4 \%$ paraformaldehyde at $4^{\circ} \mathrm{C}$, cryo-protected overnight in $30 \%$ sucrose at $4^{\circ} \mathrm{C}$, snap frozen in 50:50, OCT: $30 \%$ sucrose $\operatorname{mix}$ and cryosectioned at a thickness of $10 \mu \mathrm{m}$.

\section{In situ Hybridization/Immunohistochemistry}

In situ hybridization was performed as previously described (Yu et al., 2009). The Dll1 riboprobe (Ramos et al., 2010) was labeled with digoxigenin for both chromogenic and fluorescent in situ hybridizations. The Neurog2 riboprobe (Gradwohl et al., 1996) was labeled with dinitrophenol for fluorescent in situ hybridizations. For double in situ hybridization, probes were detected sequentially and slides were incubated in $10 \mathrm{mM} \mathrm{HCl}$ prior to detection of the second probe.

For immunohistochemistry subsequent to fluorescent in situ hybridization, antigen retrieval was carried out in $10 \mathrm{mM}$ citrate buffer $\mathrm{pH} 6.0$ in a $90^{\circ} \mathrm{C}$ water bath for $40 \mathrm{~min}$. The primary antibody was rabbit anti-Tbr2 (1:100; Abcam Ab23345) and the secondary antibody was goat anti-rabbit IgG Alexa Fluor ${ }^{\circledR} 488$ (1:400; Abcam). Nuclei were counterstained with DAPI (Thermo Fisher Scientific, Waltham, MA, USA).

\section{Cell Counting}

The density of Dll1-expressing cells and total cell density were quantified using images produced from in situ hybridization and 

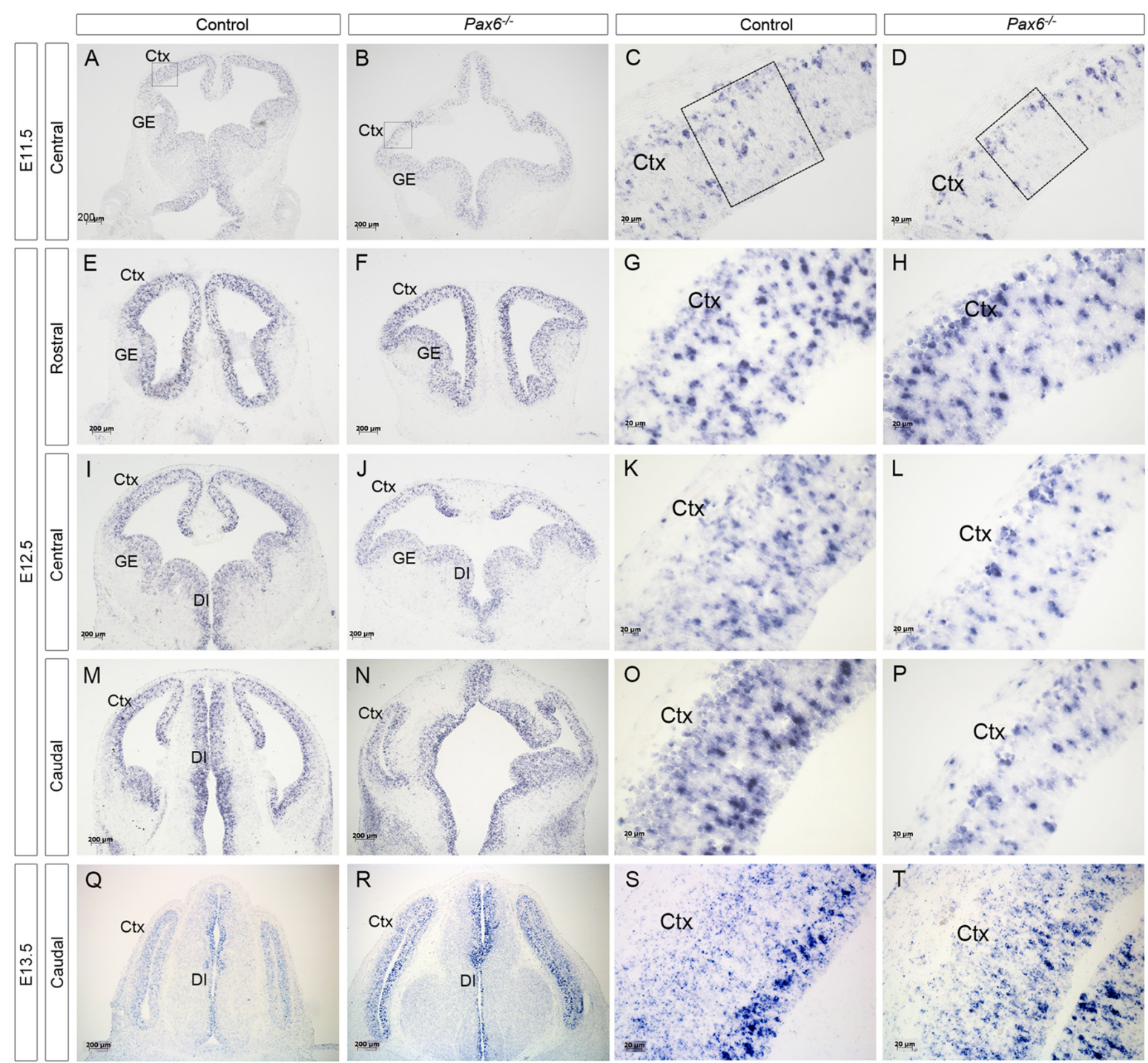

FIGURE 1 | Expression of delta-like 1 (D/l1) in Pax6 $6^{-/-}$and control forebrain between E11.5 and E13.5. In situ hybridization showing D//1 expression in Pax6 ${ }^{-/-}$ mutant and control forebrains at E11.5 (A-D), E12.5 (E-P) and E13.5 (Q-T). At E11.5, the diencephalon in Pax6 ${ }^{-/-}$mutants has a more open structure than in controls (Caballero et al., 2014) and the dorsal telencephalon is smaller, leading to the altered appearance of the section in (B). Scale bars two leftmost columns $=200 \mu \mathrm{m}$. Scale bars in rightmost columns = $20 \mu \mathrm{m}$. Abbreviations: Ctx, cortex; GE, ganglionic eminence; DI, diencephalon. Boxes in (C,D) indicate representative areas used for counting DIl1+ cells.

H\&E staining experiments. Sample areas were defined at rostral, central and caudal regions of the cortex and cells were counted in both medial and lateral areas of the cortex at each region (Figure 2A schematic). Three sections per region, per embryo were analyzed for each mutant and the appropriate control littermate. Three embryos of each genotype and developmental stage were analyzed, except for analysis of Dll1+ cell density in PAX77 embryos at E12.5 where five embryos were used.

The width of the cortex at each area counted was measured and a square was drawn on the tissue with each side equating to the recorded width, to create the sample area for counting. The total number of cells within each sample area was counted then divided by the area to give the cell density, expressed as number of cells per $0.01 \mathrm{~mm}^{2}$. The average cell density for each region was calculated and statistical analysis by two or three-way analyses of variance (ANOVA) and Tukey's multiple comparison test was conducted.

Cell count analysis was carried out using ImageJ software and statistical analysis was carried out using Prism 6 software.

\section{RESULTS}

\section{Expression of DII1 in $\mathrm{Pax6}^{-/-}$Mutant Embryonic Cortex}

Previous analysis of the cortical transcriptome of $\mathrm{Pax}^{-/-}$ mutant embryos revealed that overall Dll1 expression is reduced 
compared to controls (Sansom et al., 2009). To determine how absence of Pax6 affects Dll1 expression in the embryonic cortex in more detail, we carried out in situ hybridization for Dll1 on $\mathrm{Pax6}^{-/-}$mutant and control embryos between E11.5 and 13.5 (Figure 1). In controls, Dll1 was expressed in a salt and pepper pattern throughout the telencephalic tissue at E11.5, E12.5 and E13.5, in agreement with previous studies (Lindsell et al., 1996; Kageyama et al., 2008; Kawaguchi et al., 2008; Shimojo et al., 2008; Yoon et al., 2008). The distinctive salt and pepper pattern is a consequence of periodic oscillations in Dll1 mRNA levels, as found for a number of Notch pathway components (Shimojo et al., 2016). Pax6 ${ }^{-/-}$embryos showed a similar salt-and-pepper pattern of Dll1 expression at all three ages (Figure 1). The density of Pax6-expressing cells appeared lower in some mutant sections (e.g., compare panels Figures 1D,L,P to Figures $\mathbf{1 C}, \mathbf{K}, \mathbf{O}$ ). To test whether Dll1 expression was altered in the Pax6 mutant embryos, we systematically counted the density of Dll1-expressing cells in age-matched mutant and control embryos. Pax6 is expressed in a gradient across the cortex (rostrolateral high to caudomedial low) so, to test for possible differential effects caused by differing levels of Pax6 expression, we calculated the density of Dll1-expressing cells in both medial and lateral areas of sections taken from rostral, central and caudal levels of $\mathrm{Pax}^{-/-}$ and control cortex at E11.5, 12.5 and 13.5 (Figure 2). At E11.5, the density of Dll1-expressing cells appeared consistently higher in lateral regions of the cortex than in equivalent medial regions, both in control and $\mathrm{Pax6}^{-/-}$embryos (Figure 2A). Two-way ANOVA analysis confirmed a significant effect of region $(p<0.001)$ and post hoc analysis by Tukey's multiple comparison test showed a significantly lower density of Dll1-expressing cells in medial than lateral cortex at rostral $(p=0.003)$, central $(p=0.015)$ and caudal $(p=0.03)$ levels in control embryos. A similar pattern was found in $\mathrm{Pax}^{-/-}$ mutants and ANOVA showed that there were no significant differences between mutant and control cortices in any of the six counted areas.

At E12.5, ANOVA showed that there were no significant regional differences in the density of Dll1-expressing cells, either in controls or $\mathrm{Pax6}^{-/-}$mutants (Figure 2B), indicating that the medial-lateral difference observed at E11.5 has disappeared by E12.5. Dll1-expressing cell density was lower in all regions of $\mathrm{Pax}^{-/-}$cortex compared to controls (Figure 2B) and ANOVA indicated a significant effect of genotype $(p<0.001)$. Post hoc analysis (using Tukey's multiple comparison test) confirmed significant decreases in the density of Dll1-expressing cells in $\mathrm{Pax6}^{-/-}$mutants in both centrolateral and caudomedial cortex $(p=0.028$ and $<0.0001$, respectively), but the decreases in other regions were not significant.

At E13.5, two-way ANOVA showed significant effects of both region and genotype (both $p<0.001$ ). In control embryos, Dll1-expressing cell density was highest in caudomedial cortex and post hoc analysis (Tukey) showed that Dll1-expressing cell density in caudomedial cortex was significantly higher than in rostrolateral $(p=0.0006)$ and centrolateral cortex $(p<0.0001)$ but differences to other regions were not significant (Figure 2C).

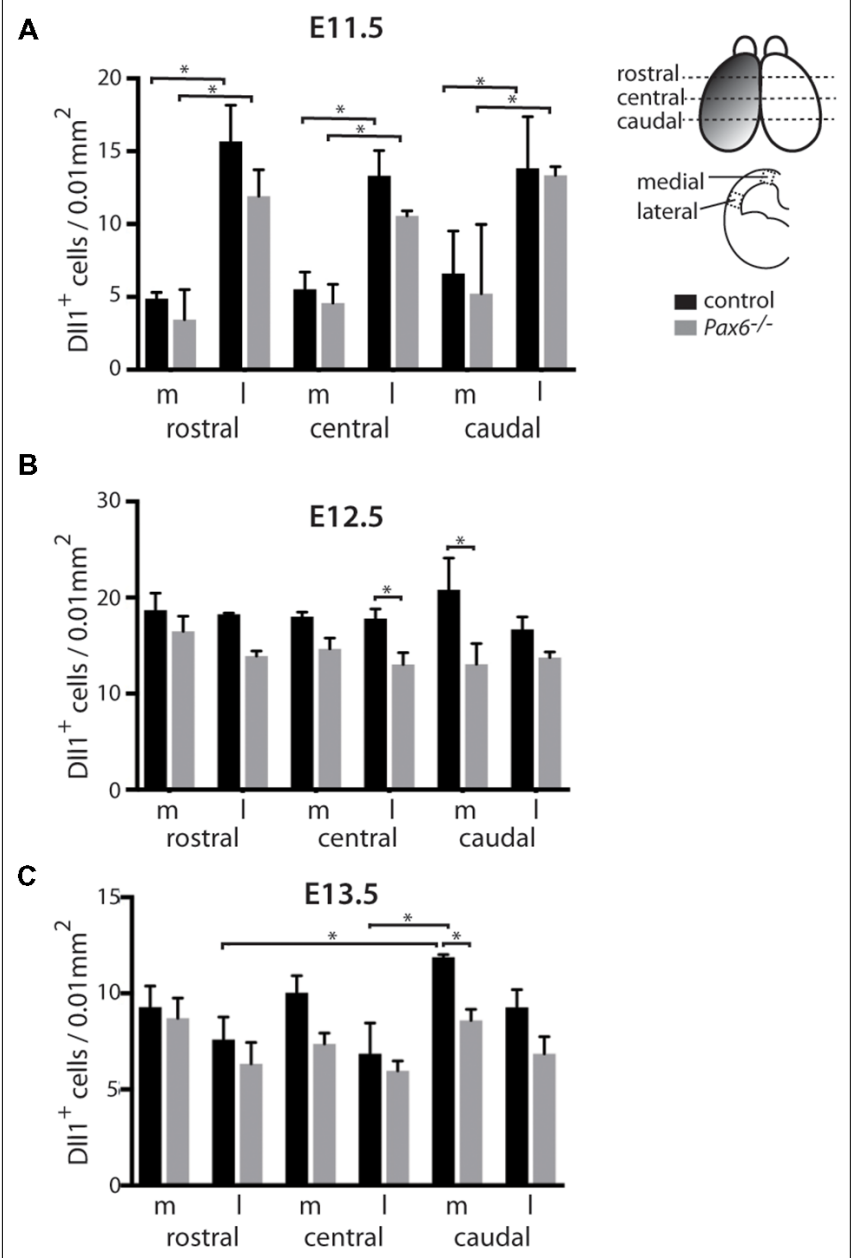

FIGURE 2 | Quantitation of DII1 + cells in Pax6 ${ }^{-/-}$mutant and control cortex at E11.5, E12.5 and E13.5. Bar graphs showing the density of Dll1-expressing cells in specific regions of the cortex in $\mathrm{Pax}^{-/-}$mutant and control embryos at E11.5 (A) E12.5 (B) and E13.5 (C). The schematic diagram in the top right corner indicates approximate positions from which samples were taken for counting. Shading on the left hemisphere indicates the gradient of Pax 6 expression. $N=3$ embryos of each genotype at each age. ${ }^{*} p<0.05$, Tukey's multiple comparison test.

Post hoc analysis revealed that these regional differences were not present in $\mathrm{Pax6}^{-/-}$mutants (Figure 2C). We found a significant decrease in Dll1-expressing cell density in caudomedial cortex of $\mathrm{Pax6}^{-1-}$ mutants when compared to the same region in controls $(p=0.012)$, but there was no significant difference between mutant and control embryos in any of the other regions tested (Figure 2C).

We found no significant differences in the overall cell density between control and mutant cortex at E11.5 $\left(82.3 \pm 6.8\right.$ vs. $89.7 \pm 15.2$ cells $\left./ 0.01 \mathrm{~mm}^{2}\right)$, E $12.5(101.3 \pm 4.4$ vs. $101 \pm 8.8$ cells $\left./ 0.01 \mathrm{~mm}^{2}\right)$ or E13.5 (111 \pm 6.3 vs. $108 \pm 8.1$ cells $/ 0.01 \mathrm{~mm}^{2}$ ). This indicates that the observed differences in the density of Dll1-expressing cells in Pax6 ${ }^{-/-}$ cortex are due to changes in the number of Dll1-expressing cells, and not simply a consequence of a lower total number of cells in Pax6 $^{-/-}$mutants. 
These findings suggest that Pax6 loss lowers the proportions of Dll1-expression produced in caudomedial regions but leaves open the possibility that it also affects production in other area. To examine this further, we switched from constitutive to conditional mutants.

\section{Conditional Mutation of Pax6 Causes a Widespread Decrease in DII1 Expression}

As Pax6 is expressed throughout cortical development, from around E8.5, it is possible that our results on the changes in Dll1 expression in $\mathrm{Pax6}^{-/-}$embryos are complicated by the effects of indirect, downstream consequences of the absence of Pax6 activity at earlier stages, including the possibility of compensatory adaptations. We therefore tested the effects of acute, conditional deletion of Pax6 on cortical expression of Dll1. Inducible loss of Pax6 was achieved by combining a tamoxifen-inducible Emx1-creERT2 transgene that is expressed specifically in cortex (Kessaris et al., 2006) with a conditional (floxed) allele of Pax6 (Simpson et al., 2009). The genotype of the experimental animals was Emx1CreEr ${ }^{T 2}$; $P a x 6^{\operatorname{lox} P / \operatorname{lox} P}$ (referred to as $\mathrm{CKO}$ ) and heterozygous littermates (i.e., $E m \times 1 \mathrm{CreEr}^{\mathrm{T}} ; \mathrm{Pax}^{\mathrm{loxP} /+}$ ) were used as controls. Our previous work with this strain showed that Pax6 protein is still present at E11.5 when tamoxifen is administered at E9.5 $(\mathrm{Mi}$ et al., 2013), so we confined our analysis of the cKO strain to E12.5 and E13.5. Both cKO and control mutant embryos expressed Dll1 in a distinctive salt and pepper pattern at E12.5 and E13.5, similar to that seen in control and $\mathrm{Pax6}^{-/-}$ embryos (Figure 3).
Quantitation revealed a lower mean density of Dll1expressing cells across E12.5 cKO mutant cortex compared to controls (Figure 4A). Two-way ANOVA showed a significant effect of genotype $(p<0.0001)$ and post hoc analysis by Tukey's multiple comparison test confirmed significant decreases in Dll1-expressing cell density in cKO cortex at rostromedial $(p=0.003)$, rostrolateral $(p=0.01)$, centromedial $(p=0.002)$ and caudomedial $(p=0.005)$ levels. Dll1 + cell density was also lower in centrolateral and caudolateral cKO cortex, but the differences were not significant ( $p=0.08, p=0.99$ respectively). Thus, conditional inactivation of Pax6 caused a widespread significant reduction in the density of Dll1-expressing cells in E12.5 cortex.

At E13.5, the mean densities of Dll1-expressing cell were lower in cKO cortex than in control embryos (Figure 4B). Two-way ANOVA confirmed a significant effect of genotype $(p<0.0001)$ but post hoc analysis (Tukey) indicated that only the caudomedial region of $\mathrm{cKO}$ mutant cortex showed a significant reduction in Dll1 expression at this stage $(p=0.012)$. Overall, conditional mutagenesis had a greater and more widespread effect than constitutive Pax6 loss, suggesting that Pax6 is required during corticogenesis for the production of a normal complement of Dll1-expressing cells across cortical regions irrespective of the regional variation in normal levels of Pax6 expression.

\section{Overexpression of Pax6 Has No Effect on Density of DII1-Expressing Cells}

To further test whether the production of Dll1-expressing cells is sensitive to the level of Pax6 expression, we examined embryos
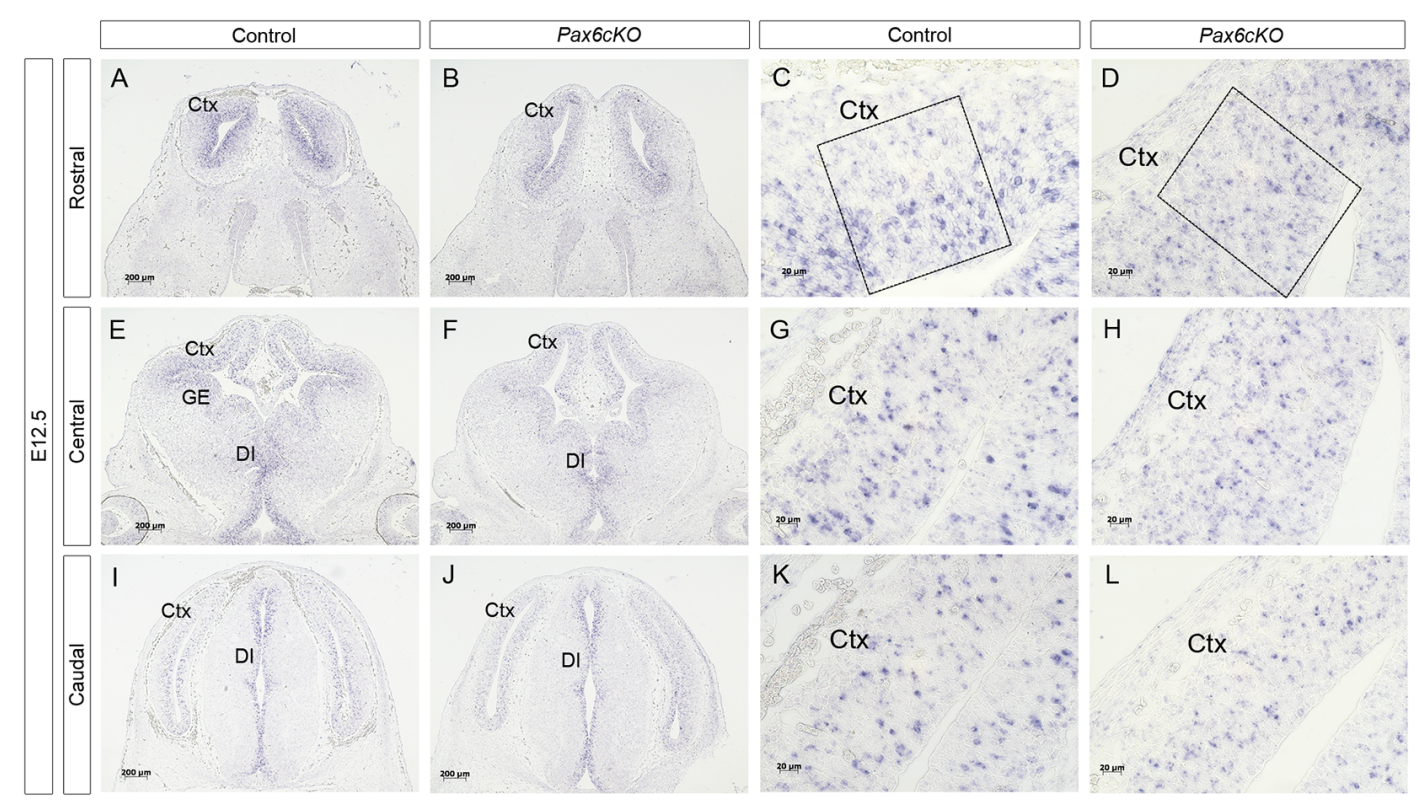

FIGURE 3 | Expression of DII1 in Pax6 cKO mutant forebrain at E12.5. In situ hybridization showing DII1 gene expression in rostral (A-D), central (E-H) and caudal (I-L) regions of E12.5 Pax6 conditional mutant and control embryonic forebrains. Panels (C,G,K) show higher power images of regions shown in (A,E,I) and panels

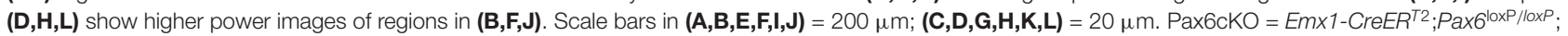
control $=E m \times 1-C r e E R^{T 2} ; P a x 6^{\text {loxP } /+}$. Abbreviations: Ctx, cortex; GE, ganglionic eminence, DI, diencephalon. Boxes in (C,D) indicate representative areas used for counting DII1+ cells. 


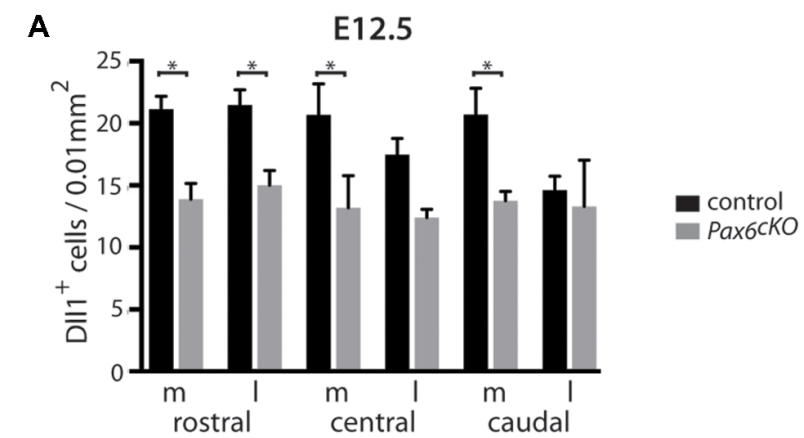

B

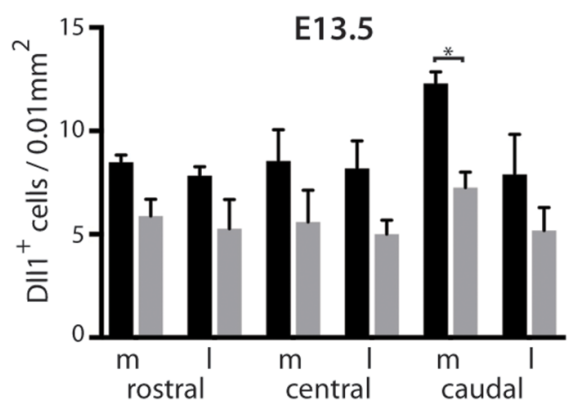

FIGURE 4 | Quantitation of DII1+ cells in Pax6 cKO and control forebrain at E12.5 and E13.5. Bar graphs comparing the density of D/11-expressing cells in specific regions of the cortex in Pax6 $\mathrm{cKO}$ mutant and control embryos at E12.5 (A) and E13.5 (B). N = 3 embryos of each genotype at each age. ${ }^{*} p<0.05$, Tukey's multiple comparison test.

from the $P A X 77$ transgenic mouse strain, which expresses up to three-fold higher levels of PAX6 protein (Manuel et al., 2007). No obvious effect of position or genotype on Dll1 expression was evident (Figures 5A-C). Two-way ANOVA analysis confirmed no significant effect of genotype at E11.5 or E13.5 ( $p=0.35$, 0.57 respectively) but did indicate an effect of genotype at E12.5 ( $p=0.008)$. However, post hoc analysis by Tukey's multiple comparison test showed no significant difference between the density of Dll1-expressing cells in PAX77 and control cortex at any of the six cortical regions examined. This supports the hypothesis that, while Pax6 is required for the production of a normal complement of Dll1-expressing cells, its levels do not affect the numbers produced.

\section{No Apparent Co-expression of DIl1 and Tbr2 in Developing Cortex}

Previous studies have shown that loss of Pax6 causes a loss of Tbr2+ IPCs (Quinn et al., 2007). We assessed whether Tbr2+ IPCs express Dll1 RNA since, if they do, our findings in Pax6 loss-of-function mutants might be explained by a loss of Tbr2+ IPCs. We combined in situ hybridization for Dll1 with immunohistochemistry for the IPC marker Tbr2 in E12.5 and E14.5 cortex (Figure 6). Strong Tbr2 expression was seen in control embryos at E12.5, spanning the length of the cortex and largely confined to the subventricular zone (SVZ; Figures 6A,C). In contrast, Dll1 expression was largely restricted to the VZ. We
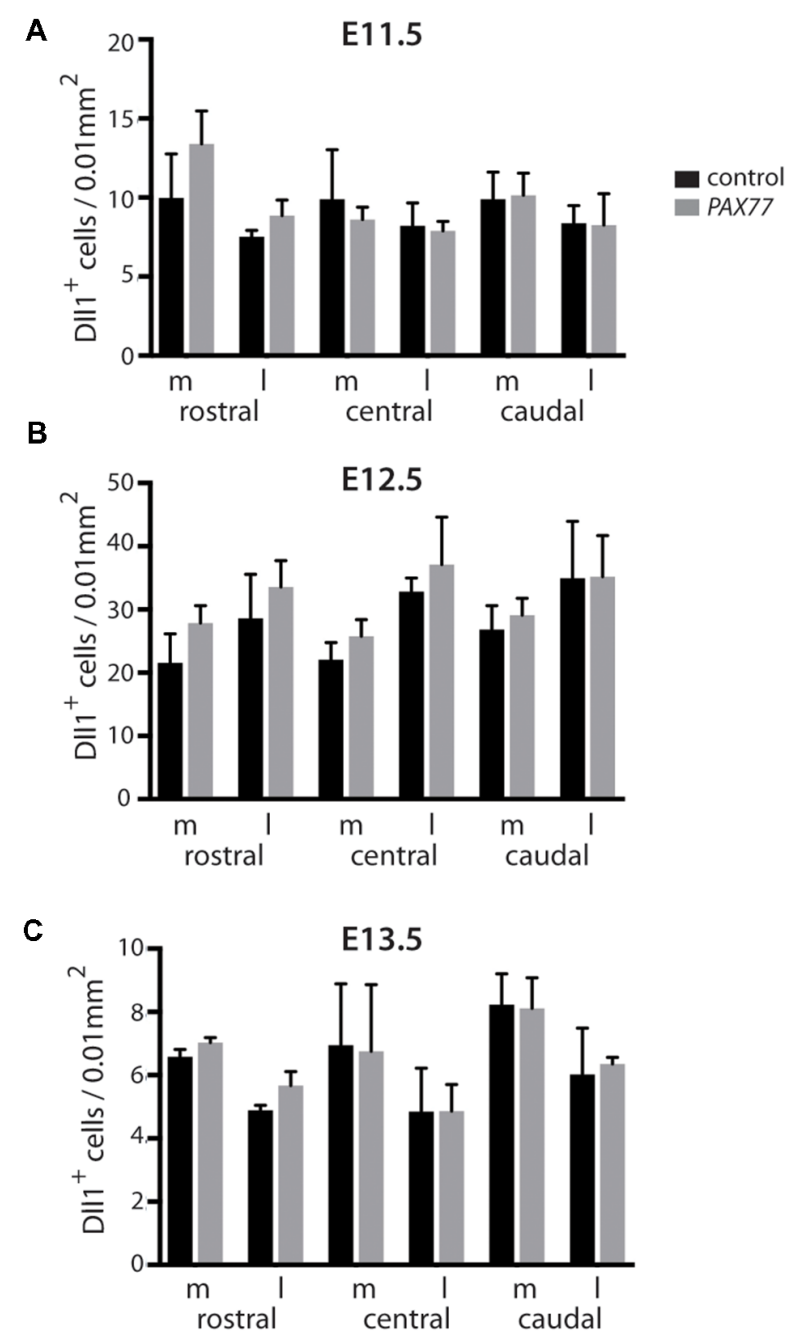

FIGURE 5 | Quantitation of D/l1 + cells in PAX77 and control forebrain at E11.5, E12.5 and E13.5. Bar graphs show the density of DI/1-expressing cells in specific regions of the cortex in PAX77 transgenic and control embryos at E11.5 (A), E12.5 (B) and E13.5 (C). No statistically significant changes were found (analysis of variance, ANOVA). $N=3$ embryos of each genotype at each age.

found no evidence of co-expression of Dll1 and Tbr2 in control cortex at E12.5 (Figures 6A,C), indicating that a clear majority of Tbr2+ cells do not express Dll1. As reported previously, the number of Tbr2-expressing cells was greatly reduced in $\mathrm{Pax}^{-/-}$ mutants at E12.5 (Figures 6B,D, Quinn et al., 2007). As in controls, no evidence of co-expression of Dll1 and Tbr2 was found in the $\mathrm{Pax}^{-/-}$cortex (Figures 6B,D).

At E14.5, the SVZ constitutes the majority of the cortical progenitor zone. Dll1 expression remained largely confined to the narrower $\mathrm{VZ}$ region (Figures $\mathbf{6 E}, \mathbf{G}, \mathbf{I}, \mathbf{K}$ ). As at E12.5, no co-localization between Dll1 and Tbr2 was observed (Figures 6G,I,K). Interestingly, however, strong co-localization of Dll1 and Tbr2 was found in the developing hippocampus (HP) region at E14.5 (Figure 6E, region between white arrowheads), indicating that many IPCs in the HP express Dll1, in contrast to 


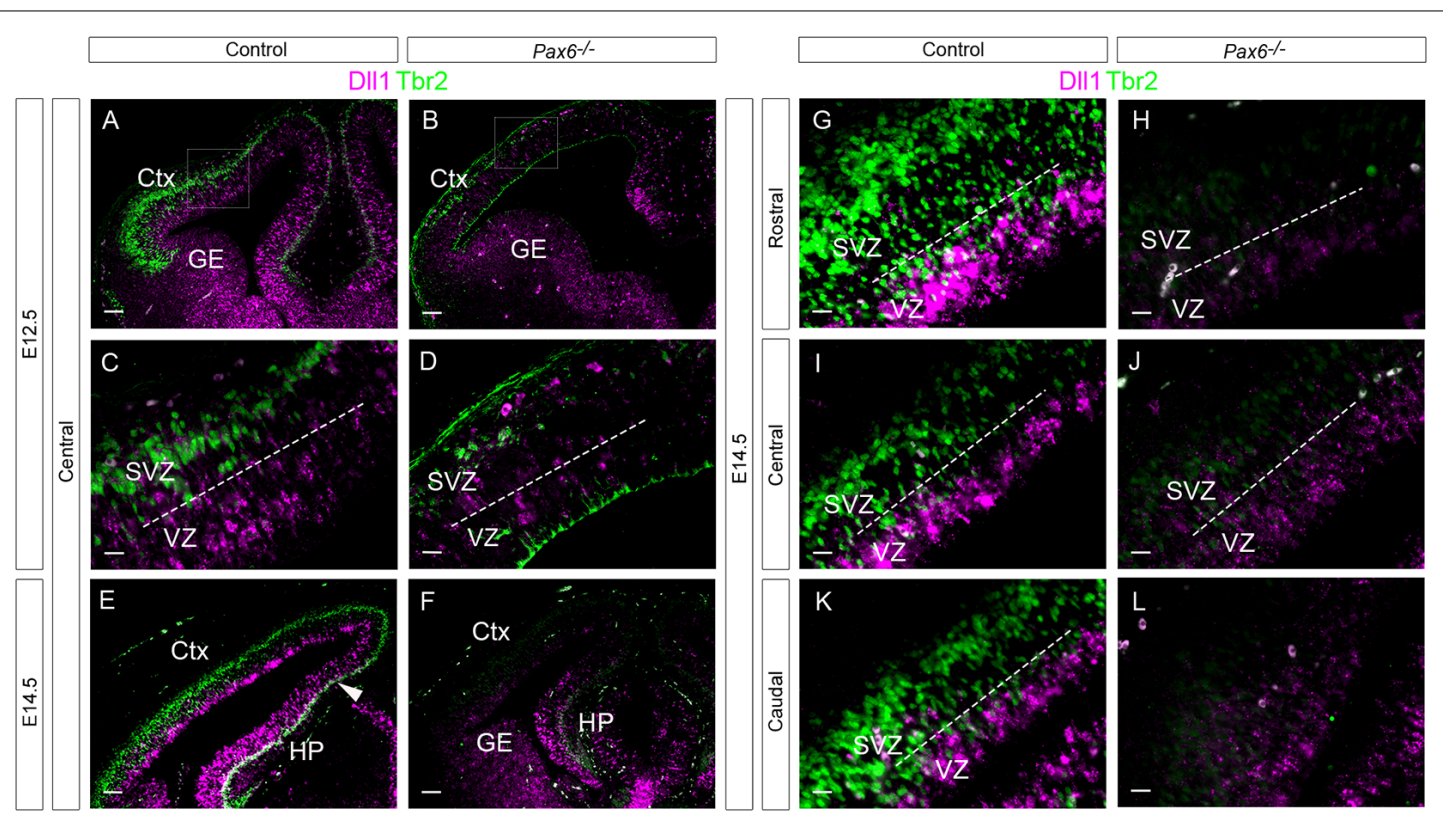

FIGURE 6 | Expression of DII1 and Tbr2 in Pax6 ${ }^{-/-}$and control embryos at E12.5 and E14.5. In situ hybridization for DI/1 (magenta) and immunohistochemistry for Tbr2 (green) at E12.5 (A-F) and E14.5 (G-L). The area delineated by arrowheads in (E,F) indicates the presumptive hippocampal region, where DII1 and Tbr2 are co-expressed. Dotted lines indicate the boundary between the VZ and the SVZ. Abbreviations: Ctx, cortex; GE, ganglionic eminences; HP, hippocampus; VZ, ventricular zone; SVZ, subventricular zone. Scale bars (A,B,E,F): $80 \mu \mathrm{m}$; (C,D,G-L): $20 \mu \mathrm{m}$.

the neocortex. The presence of Dll1 expression in both the VZ and SVZ of the developing HP suggests that it is expressed by both RGCs and IPCs in this region.

In E14.5 Pax6 $^{-/-}$mutant embryos, we found no cells co-expressing both Dll1 and Tbr2 in either the cortex or the HP (Figure 6F). As at E12.5, there were many fewer Tbr2-expressing cells in E14.5 Pax6 $6^{-/-}$mutants than in controls (Figures 6F,H,J,L).

These findings suggest that the loss of Dll1-expressing cells in Pax6 loss-of-function mutants is not a manifestation of the reduction of Tbr2+ IPCs in their cortices. We next considered whether it might be a consequence of altered Neurog2 expression.

\section{Overlapping Neurog2 and DIl1 Expression in Embryonic Cortex}

As Pax6 can activate expression of Neurog2 (Scardigli et al., 2003), and Neurog2 can drive expression of Dll1 (Castro et al., 2006), we tested the possibility that the changes in Dll1+ cell density found in Pax6 mutant cortex could be mediated through Neurog2. We first compared the expression of Neurog2 and Dll1 in Pax6 $6^{-/-}$mutants and controls, to test whether cortical progenitor cells express both Neurog2 and Dll1. At E12.5, Dll1 and Neurog2 were expressed widely throughout the progenitor zone of the developing cerebral cortex in control embryos and many Neurog2/Dll1 double positive cells were evident (Figures 7A,C) consistent with the possibility that Pax6 could regulate Dll1 through Neurog2. In Pax6 ${ }^{-/-}$ mutants, there were substantially fewer Neurog2-expressing cells, especially in the lateral cortex (Figures 7B,D) and, in contrast to controls, few Dll1 Neurog2 double-positive cells were seen in

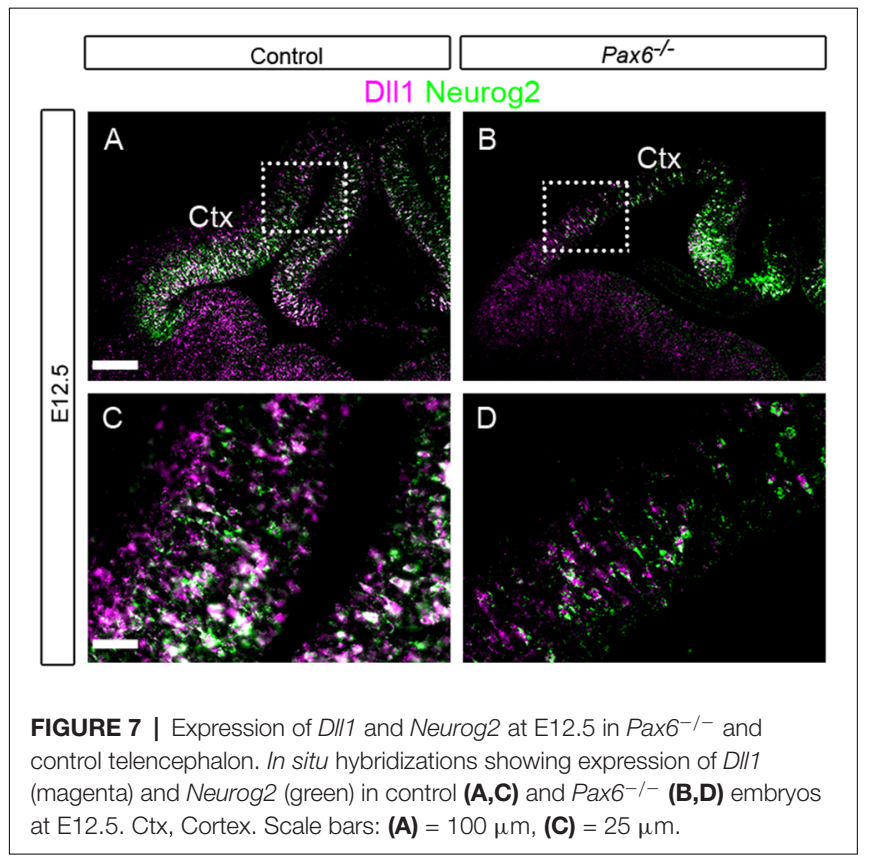

$\mathrm{Pax6}^{-/-}$mutant cortex (Figure 7D), indicating that $\mathrm{Pax6}^{-/-}$ cells that lacked expression of Neurog2 were nonetheless able to express Dll1.

\section{DII1 Expression Is Unaffected in Neurog2-/- Mutant Cortex}

Our hypothesis that Pax6 regulates Dll1 expression through Neurog2 predicts that decreased expression of Neurog2 should 

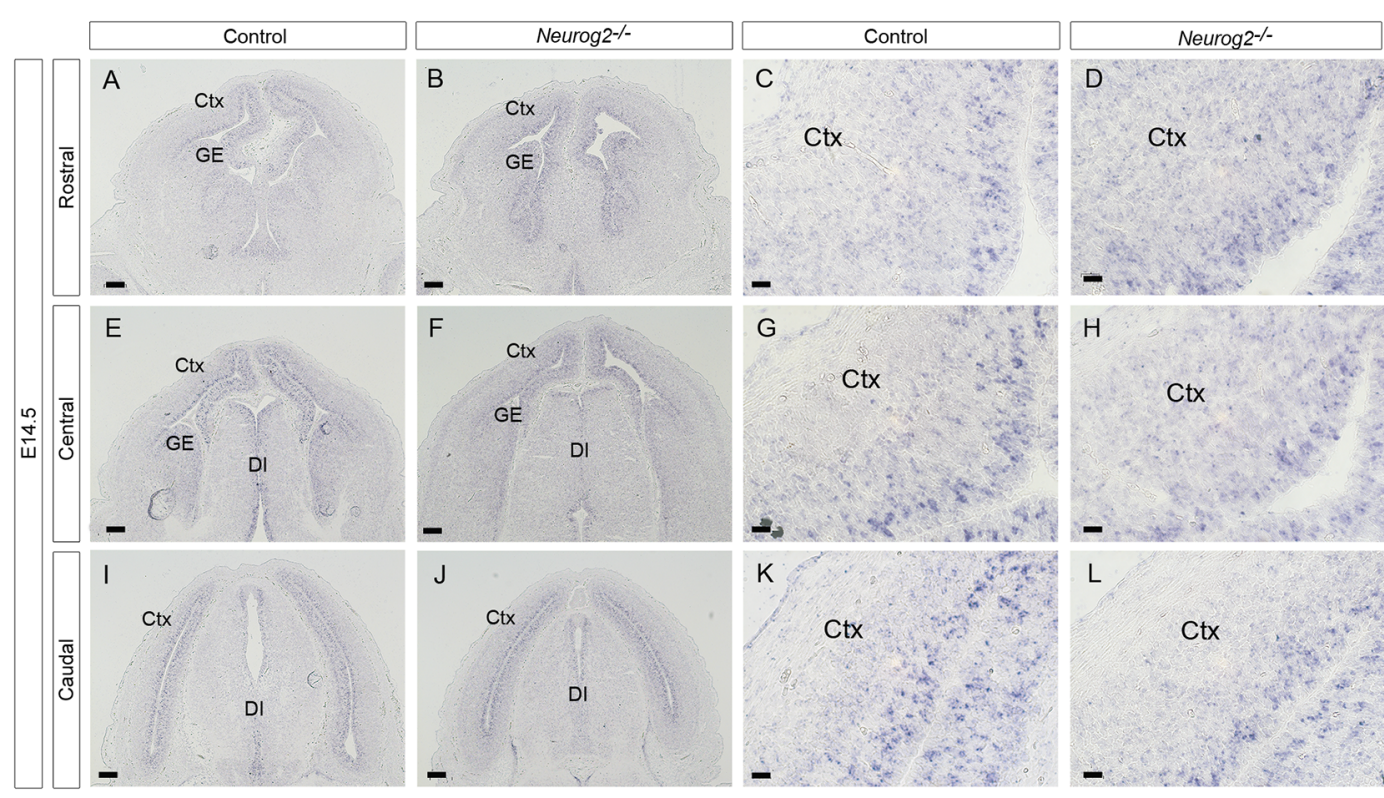

FIGURE 8 | D/l1 expression in E14.5 Neurog2-/- mutant embryos. In situ hybridization showing D/l1 expression in rostral (A,B), middle (E,F) and caudal (I,J) regions of E14.5 Neurog2 ${ }^{-/-}$and control embryonic forebrains. Panels $\mathbf{( C , G , K )}$ and $\mathbf{( D , H , L )}$ show higher power images of regions shown in $\mathbf{( A , E , I ) ~ a n d ~} \mathbf{( B , F , J ) ~}$ respectively.

lead to lower Dll1 expression. However, while Pax6 mutants showed substantially decreased Neurog2 expression, the effect on Dll1 was less pronounced (Figure 7). To test directly whether Neurog2 regulates Dll1 expression, we generated homozygous Neurog $2^{-/-}$mutant embryos by intercrossing heterozygous animals carrying a Neurog2 allele which lacks Neurog2 activity as a result of the insertion of cre recombinase coding sequence (Zirlinger et al., 2002). Dll1 expression, as shown by in situ hybridization, appeared very similar in E14.5 Neurog $2^{-/-}$(Figures 8B,D,F,H,J,L) and control embryos (Figures 8A,C,E,G,I,K), each showing the familiar salt and pepper staining pattern. Quantitation of Dll1-expressing cell density in these embryos revealed no statistically significant differences between Neurog $2^{-/-}$mutants and controls (data not shown).

\section{DISCUSSION}

\section{Evidence for Interactions Between Pax6 and DIII}

To investigate a possible role for the Notch ligand Dll1 in the pathway by which Pax6 influences the balance between proliferation and differentiation of RGC progenitors in embryonic cortex, we characterized the cortical expression of Dll1 in detail during key neurogenic stages, both in control and $\mathrm{Pax6}^{-/-}$mutant embryos. In control embryos at the earliest stages of neurogenesis (E11.5), Dll1-expressing cells were significantly more abundant in lateral cortex than medial. Pax6 is expressed in a high lateral to low medial gradient, so the presence of more Dll1+ cells in lateral than medial cortex could suggest an effect of Pax6 levels on Dll1 expression. However, the medial-lateral difference in Dll1 expression was also seen in E11.5 Pax6 ${ }^{-/-}$embryos and we found no differences in Dll1+ cell density between $\mathrm{Pax}^{-/-}$mutant and control embryos at any position in the E11.5 cortex, arguing against a direct role for Pax6 levels in setting up the medio-lateral difference in Dll1+ cell density.

At E12.5, the medial-lateral difference in Dll1 expression was no longer evident. In controls, Dll1+ cell density was highest in caudomedial cortex, but this was not significantly different to any other region. In E12.5 $\mathrm{Pax6}^{-/-}$mutants, Dll1 expression was decreased in centrolateral and caudomedial areas of cortex compared to controls, but unaffected in other regions. In E13.5 control embryos, caudomedial cortex again showed the highest density of Dll1+ cells, although this was significantly higher than only centrolateral and mediolateral areas. In E13.5 Pax $^{-/-}$mutants Dll1 + cells were again significantly reduced in caudomedial cortex, but all other regions showed no significant change in Dll1+ cells at this stage. Perhaps surprisingly, the region which showed the most consistent changes in Dll1+ cells in Pax6 mutants, caudomedial cortex, is also the region where Pax6 expression levels are lowest.

Dll1 expression was more strongly affected in conditional Pax6 mutants. Fewer Dll1+ cells were found throughout almost all regions of the E12.5 cortex in cortex-specific Pax6 $^{-/-}$mutant embryos, in contrast to constitutive $\mathrm{Pax}^{-/-}$mutants, in which only centrolateral and caudomedial cortex showed significant differences. By E13.5 only caudomedial cortex was affected, the same region affected in constitutive $P a x 6^{-/-}$embryos at this stage. A likely explanation for this difference could be the presence of a mechanism which can compensate for the loss of Pax6 in all except caudomedial cortex in constitutive Pax6 ${ }^{-/-}$ 
mutants but in the E12.5 cKO mutants has not had enough time after loss of Pax6 for the compensation mechanism to act, such that the effect of Pax6 loss is seen throughout the cortex. The principle that conditional mutants may have more severe phenotypes than constitutive mutants is well established (Rossi et al., 2015). Increasing the level of Pax6 had no significant effect on Dll1+ cell numbers, consistent with the finding that gain of Pax6 function generally has a milder effect on cortical development than does loss of function (Manuel et al., 2007; Georgala et al., 2011).

We found large numbers of Dll1+ cells in the VZ of mouse embryonic cortex, many of them co-expressing Neurog2, showing that Dll1 is widely expressed in cortical progenitors. However, we did not find any evidence of Dll1/Tbr2 co-expressing cells, suggesting that Dll1 mRNA expression in Tbr2+ basal progenitor cells is less common. Dll1 protein has been detected in Tbr2+ basal progenitors (Nelson et al., 2013), suggesting that the protein persists after Dll1 mRNA is no longer expressed. Indeed, Dll1 protein produced by basal progenitors has been suggested to activate Notch signaling in radial glial progenitors in the VZ (Mizutani et al., 2007; Yoon et al., 2008; Nelson et al., 2013).

\section{How Might Pax6 Regulate DIl1 Expression?}

We found that loss of Pax6 leads to a decrease in the number of Dll1+ cells in specific areas of the cortex during neurogenesis. The absence of a straightforward relationship between Pax6 function and expression levels and Dll1 expression suggests that the interaction between these genes is likely to be indirect. We investigated the possibility that Pax6 could exert its effect on Dll1 expression indirectly, through the Pax6-regulated neurogenic transcription factor Neurog2 which can regulate Dll1 (Castro et al., 2006). In support of this possibility, many embryonic cortical progenitor cells express both Neurog2 and Dll1 and expression of both of these genes is decreased in Pax6 $6^{-/-}$mutants. However, no significant difference in Dll1 expression was observed in Neurog $2^{-/-}$cortex, indicating that the mechanism is not as simple as this. The continued Dll1 expression in Neurog $2^{-/-}$mutants suggests that the absence of Neurog2 can be compensated for. The most likely candidates to mediate such compensation are the related neurogenic genes Neurog1 and Ascl1. Neurog1 and Neurog2 are both normally

\section{REFERENCES}

Caballero, I. M., Manuel, M. N., Molinek, M., Quintana-Urzainqui, I., Mi, D., Shimogori, T., et al. (2014). Cell-autonomous repression of Shh by transcription factor Pax6 regulates diencephalic patterning by controlling the central diencephalic organizer. Cell Rep. 8, 1405-1418. doi: 10.1016/j.celrep.2014. 07.051

Castro, D. S., Skowronska-Krawczyk, D., Armant, O., Donaldson, I. J., Parras, C., Hunt, C., et al. (2006). Proneural bHLH and Brn proteins coregulate a neurogenic program through cooperative binding to a conserved DNA motif. Dev. Cell 11, 831-844. doi: 10.1016/j.devcel.2006.10.006

Elsen, G. E., Bedogni, F., Hodge, R. D., Bammler, T. K., MacDonald, J. W., Lindtner, S., et al. (2018). The epigenetic factor landscape of developing neocortex is regulated by transcription factors Pax $6 \rightarrow$ Tbr $2 \rightarrow$ Tbr1. Front Neurosci. 12:571. doi: 10.3389/fnins.2018.00571 expressed throughout the cortex, whereas Ascl1 expression is largely confined to ventral telencephalon (Fode et al., 2000). In Neurog $2^{-/-}$mutants, cortical expression of Neurog1 is decreased slightly, but Ascl1 expression is strongly upregulated (Fode et al., 2000). The phenotype of $A s c l 1^{-/-}$; Neurog2 $2^{-/-}$double mutants is much more severe than that of Neurog $2^{-/-}$single mutants, indicating that Ascl1 compensates for effects of loss of Neurog2 function (Fode et al., 2000). Further, Ascl1 has been shown to be able to directly activate Dll1 expression, acting on a different enhancer element to that activated by Neurog2 (Castro et al., 2006). Therefore, increased levels of Ascl1 in Neurog $2^{-/-}$cortex may maintain expression of Dll1 at normal levels. However, Ascl1 is also upregulated in $\mathrm{Pax6}^{-/-}$mutant embryonic cortex (Kroll and O'Leary, 2005), yet significant regional decreases in Dll1+ cells are observed. This could be consistent with a direct role for Pax6 in regulating Dll1, that cannot be compensated for by Ascl1, or may indicate that the regulation of Dll1 is complex and that other factors are involved.

\section{DATA AVAILABILITY}

All datasets generated for this study are included in the manuscript.

\section{AUTHOR CONTRIBUTIONS}

ED designed and performed experiments and data analysis. ED, DP and JM discussed experimental design and results and wrote the manuscript. All authors read and approved the final manuscript.

\section{FUNDING}

This research was funded by Medical Research Council (MRC) grant N012291/1 and Biotechnology and Biological Sciences Research Council (BBSRC) grant N006542/1 and a BBSRC PhD studentship to ED.

\section{ACKNOWLEDGMENTS}

We are grateful to Gabriella Clarke for her contribution to this work.
Fode, C., Ma, Q., Casarosa, S., Ang, S. L., Anderson, D. J., and Guillemot, F. (2000). A role for neural determination genes in specifying the dorsoventral identity of telencephalic neurons. Genes Dev. 14, 67-80. doi: 10.1101/gad.14.1.67

Georgala, P. A., Manuel, M., and Price, D. J. (2011). The generation of superficial cortical layers is regulated by levels of the transcription factor Pax6. Cereb. Cortex 21, 81-94. doi: 10.1093/cercor/bhq061

Gradwohl, G., Fode, C., and Guillemot, F. (1996). Restricted expression of a novel murine atonal-related bHLH protein in undifferentiated neural precursors. Dev. Biol. 180, 227-241. doi: 10.1006/dbio.1996.0297

Haubensak, W., Attardo, A., Denk, W., and Huttner, W. B. (2004). Neurons arise in the basal neuroepithelium of the early mammalian telencephalon: a major site of neurogenesis. Proc. Natl. Acad. Sci. U S A 101, 3196-3201. doi: 10.1073/pnas.0308600100

Hill, R. E., Favor, J., Hogan, B. L., Ton, C. C., Saunders, G. F., Hanson, I. M., et al. (1991). Mouse small eye results from mutations in a paired-like 
homeobox-containing gene. Nature 354, 522-525. doi: 10.1038/35 $4522 \mathrm{a} 0$

Kageyama, R., Ohtsuka, T., Shimojo, H., and Imayoshi, I. (2008). Dynamic Notch signaling in neural progenitor cells and a revised view of lateral inhibition. Nat. Neurosci. 11, 1247-1251. doi: 10.1038/nn.2208

Kawaguchi, D., Yoshimatsu, T., Hozumi, K., and Gotoh, Y. (2008). Selection of differentiating cells by different levels of delta-like 1 among neural precursor cells in the developing mouse telencephalon. Development 135, 3849-3858. doi: $10.1242 /$ dev.024570

Kessaris, N., Fogarty, M., Iannarelli, P., Grist, M., Wegner, M., and Richardson, W. D. (2006). Competing waves of oligodendrocytes in the forebrain and postnatal elimination of an embryonic lineage. Nat. Neurosci. 9, 173-179. doi: 10.1038/nn1620

Kroll, T. T., and O'Leary, D. D. (2005). Ventralized dorsal telencephalic progenitors in Pax6 mutant mice generate GABA interneurons of a lateral ganglionic eminence fate. Proc. Natl. Acad. Sci. U S A 102, 7374-7379. doi: 10.1073/pnas.0500819102

Lindsell, C. E., Boulter, J., diSibio, G., Gossler, A., and Weinmaster, G. (1996). Expression patterns of Jagged, Delta1, Notch1, Notch2, and Notch3 genes identify ligand-receptor pairs that may function in neural development. Mol. Cell. Neurosci. 8, 14-27. doi: 10.1006/mcne.1996.0040

Manuel, M., Georgala, P. A., Carr, C. B., Chanas, S., Kleinjan, D. A., Martynoga, B., et al. (2007). Controlled overexpression of Pax6 in vivo negatively autoregulates the Pax6 locus, causing cell-autonomous defects of late cortical progenitor proliferation with little effect on cortical arealization. Development 134, 545-555. doi: 10.1242/dev.02764

Manuel, M. N., Mi, D., Mason, J. O., and Price, D. J. (2015). Regulation of cerebral cortical neurogenesis by the Pax6 transcription factor. Front. Cell. Neurosci. 9:70. doi: 10.3389/fncel.2015.00070

Mi, D., Carr, C. B., Georgala, P. A., Huang, Y.-T., Manuel, M. N., Jeanes, E., et al. (2013). Pax6 exerts regional control of cortical progenitor proliferation via direct repression of Cdk6 and hypophosphorylation of pRb. Neuron 78, 269-284. doi: 10.1016/j.neuron.2013.02.012

Mizutani, K., Yoon, K., Dang, L., Tokunaga, A., and Gaiano, N. (2007). Differential Notch signalling distinguishes neural stem cells from intermediate progenitors. Nature 449, 351-355. doi: 10.1038/nature06090

Nelson, B. R., Hodge, R. D., Bedogni, F., and Hevner, R. F. (2013). Dynamic interactions between intermediate neurogenic progenitors and radial glia in embryonic mouse neocortex: potential role in Dll1-Notch signalling. J. Neurosci. 33, 9122-9139. doi: 10.1523/JNEUROSCI.0791-13.2013

Noctor, S. C., Martínez-Cerdeño, V., Ivic, L., and Kriegstein, A. R. (2004). Cortical neurons arise in symmetric and asymmetric division zones and migrate through specific phases. Nat. Neurosci. 7, 136-144. doi: 10.1038/nn1172

Pierfelice, T., Alberi, L., and Gaiano, N. (2011). Notch in the vertebrate nervous system: an old dog with new tricks. Neuron 69, 840-855. doi: 10.1016/j.neuron. 2011.02.031

Pontious, A., Kowalczyk, T., Englund, C., and Hevner, R. F. (2008). Role of intermediate progenitor cells in cerebral cortex development. Dev. Neurosci. 30, 24-32. doi: 10.1159/000109848

Quinn, J. C., Molinek, M., Martynoga, B. S., Zaki, P. A., Faedo, A., Bulfone, A., et al. (2007). Pax6 controls cerebral cortical cell number by regulating exit from the cell cycle and specifies cortical cell identity by a cell autonomous mechanism. Dev. Biol. 302, 50-65. doi: 10.1016/j.ydbio.2006.08.035

Quintana-Urzainqui, I., Kozić, Z., Mitra, S., Tian, T., Manuel, M., Mason, J. O., et al. (2018). Tissue-specific actions of Pax6 on proliferation and differentiation balance in developing forebrain are Foxg1-dependent. iScience 10, 171-191. doi: 10.1016/j.isci.2018.11.031

Ramos, C., Rocha, S., Gaspar, C., and Henrique, D. (2010). Two Notch ligands, Dll1 and Jag1, are differently restricted in their range of action to control neurogenesis in the mammalian spinal cord. PLoS One 5:e15515. doi: 10.1371/journal.pone.0015515
Rossi, A., Kontarakis, Z., Gerri, C., Nolte, H., Hölper, S., Krüger, M., et al. (2015). Genetic compensation induced by deleterious mutations but not gene knockdowns. Nature 524, 230-233. doi: 10.1038/nature14580

Sansom, S. N., Griffiths, D. S., Faedo, A., Kleinjan, D. J., Ruan, Y., Smith, J., et al. (2009). The level of the transcription factor Pax6 is essential for controlling the balance between neural stem cell self-renewal and neurogenesis. PLoS Genet. 5:e1000511. doi: 10.1371/journal.pgen.1000511

Scardigli, R., Bäumer, N., Gruss, P., Guillemot, F., and Le Roux, I. (2003). Direct and concentration-dependent regulation of the proneural gene Neurogenin 2 by Pax6. Development 130, 3269-3281. doi: 10.1242/dev.00539

Schedl, A., Ross, A., Lee, M., Engelkamp, D., Rashbass, P., van Heyningen, V., et al. (1996). Influence of PAX6 gene dosage on development: overexpression causes severe eye abnormalities. Cell 86, 71-82. doi: 10.1016/s0092-8674(00)80078-1

Shimojo, H., Isomura, A., Ohtsuka, T., Kori, H., Miyachi, H., and Kageyama, R. (2016). Oscillatory control of Delta-like1 in cell interactions regulates dynamic gene expression and tissue morphogenesis. Genes Dev. 30, 102-116. doi: $10.1101 / \operatorname{gad} .270785 .115$

Shimojo, H., Ohtsuka, T., and Kageyama, R. (2008). Oscillations in notch signaling regulate maintenance of neural progenitors. Neuron 58, 52-56. doi: 10.1016/j. neuron.2008.02.014

Simpson, T. I., Pratt, T., Mason, J. O., and Price, D. J. (2009). Normal ventral telencephalic expression of Pax6 is required for normal development of thalamocortical axons in embryonic mice. Neural Dev. 4:19. doi: 10.1186/17498104-4-19

Sun, J., Rockowitz, S., Xie, Q., Ashery-Padan, R., Zheng, D., and Cvekl, A. (2015). Identification of in vivo DNA-binding mechanisms of Pax6 and reconstruction of Pax6-dependent gene regulatory networks during forebrain and lens development. Nucleic Acids Res. 43, 6827-6846. doi: 10.1093/nar/gkv589

Wolf, L. V., Yang, Y., Wang, J., Xie, Q., Braunger, B., Tamm, E. R., et al. (2009). Identification of pax6-dependent gene regulatory networks in the mouse lens. PLoS One 4:e4159. doi: 10.1371/journal.pone.0004159

Xie, Q., Yang, Y., Huang, J., Ninkovic, J., Walcher, T., Wolf, L., et al. (2013). Pax6 interactions with chromatin and identification of its novel direct target genes in lens and forebrain. PLoS One 8:e54507. doi: 10.1371/journal.pone. 0054507

Yoon, K. J., Koo, B. K., Im, S. K., Jeong, H. W., Ghim, J., Kwon, M. C., et al. (2008). Mind bomb 1-expressing intermediate progenitors generate notch signalling to maintain radial glial cells. Neuron 58, 519-531. doi: 10.1016/j.neuron.2008. 03.018

Ypsilanti, A. R., and Rubenstein, J. L. (2016). Transcriptional and epigenetic mechanisms of early cortical development: an examination of how Pax6 coordinates cortical development. J. Comp. Neurol. 524, 609-629. doi: $10.1002 /$ cne. 23866

Yu, T., Fotaki, V., Mason, J. O., and Price, D. J. (2009). Analysis of early ventral telencephalic defects in mice lacking functional Gli3 protein. J. Comp. Neurol. 512, 613-627. doi: 10.1002/cne.21918

Zirlinger, M., Lo, L., McMahon, J., McMahon, A. P., and Anderson, D. J. (2002). Transient expression of the bHLH factor neurogenin-2 marks a subpopulation of neural crest cells biased for a sensory but not a neuronal fate. Proc. Natl. Acad. Sci. U S A 99, 8084-8089. doi: 10.1073/pnas.122231199

Conflict of Interest Statement: The authors declare that the research was conducted in the absence of any commercial or financial relationships that could be construed as a potential conflict of interest.

Copyright (c) 2019 Dorà, Price and Mason. This is an open-access article distributed under the terms of the Creative Commons Attribution License (CC BY). The use, distribution or reproduction in other forums is permitted, provided the original author(s) and the copyright owner(s) are credited and that the original publication in this journal is cited, in accordance with accepted academic practice. No use, distribution or reproduction is permitted which does not comply with these terms. 